\title{
Alanine 32 in PilA is important for PilA stability and type IV pili function in Myxococcus xanthus

\begin{abstract}
Correspondence
Wenyuan Shi

wenyuan@ucla.edu
\end{abstract} \\ Received 14 March 2011 \\ Revised 11 April 2011 \\ Accepted 12 April 2011

\author{
Zhe Yang, ${ }^{1}$ Wei Hu, ${ }^{2,3}$ Kevin Chen, ${ }^{4,5}$ Jing Wang, ${ }^{2,3}$ Renate Lux, ${ }^{2}$ \\ Z. Hong Zhou ${ }^{4,5}$ and Wenyuan Shi ${ }^{1,2,5}$
} \\ ${ }^{1}$ Molecular Biology Institute, University of California, Los Angeles (UCLA), Los Angeles, CA 90095,
USA
${ }^{2}$ School of Dentistry, University of California, Los Angeles, CA 90095, USA
${ }^{3}$ State Key Laboratory of Microbial Technology, School of Life Science, Shandong University,
Jinan 250100, Shandong, PR China
${ }^{4}$ California NanoSystems Institute, UCLA, Los Angeles, CA 90095, USA
${ }^{5}$ Department of Microbiology, Immunology and Molecular Genetics, UCLA, Los Angeles,
CA 90095-7364, USA \\ Type IV pili (TFP) are membrane-anchored filaments with a number of important biological functions. In the model organism Myxococcus xanthus, TFP act as molecular engines that power social (S) motility through cycles of extension and retraction. TFP filaments consist of several thousand copies of a protein called PilA or pilin. PilA contains an N-terminal $\alpha$-helix essential for TFP assembly and a C-terminal globular domain important for its activity. The role of the PilA sequence and its structure-function relationship in TFP-dependent $S$ motility remain active areas of research. In this study, we identified an M. xanthus PilA mutant carrying an alanine to valine substitution at position 32 in the $\alpha$-helix, which produced structurally intact but retraction-defective TFP. Characterization of this mutant and additional single-residue variants at this position in PilA demonstrated the critical role of alanine 32 in PilA stability, TFP assembly and retraction.
}

\section{INTRODUCTION}

Type IV pili (TFP) are multifunctional membraneanchored filaments present in both pathogenic and nonpathogenic bacteria. An astonishing number of cellular functions have been attributed to the TFP, including adhesion, surface motility, biofilm formation, natural transformation, immune escape and cell signalling (Craig et al., 2004). TFP-mediated motility enables movement of bacterial cells across semi-solid surfaces such as mucosal epithelia and soft agar surfaces. This behaviour is called twitching motility in Pseudomonas aeruginosa and Neisseria gonorrhoeae, and social gliding motility in Myxococcus xanthus (Bradley, 1980; Kaiser, 1979).

M. xanthus is a Gram-negative soil bacterium able to glide on solid surfaces and coordinate thousands of cells into multicellular fruiting bodies during starvation. M. xanthus cells utilize two genetically distinct systems for the gliding motility to achieve their complex cell behaviours: adventurous (A) motility and social (S) motility (Hodgkin \& Kaiser, 1979). Single-cell movement via A motility is the preferred type of locomotion on dry surfaces, while

Abbreviations: EPS, extracellular polysaccharides; $S$ motility, social motility; TFP, type IV pili. coordinated movement in groups via S motility is mainly utilized on moist surfaces (Shi \& Zusman, 1993). S motility is known to be important for cell aggregation, cohesion and fruiting body morphogenesis (Hodgkin \& Kaiser, 1979; MacNeil et al., 1994). TFP in M. xanthus act as molecular engines that power S motility (Hodgkin \& Kaiser, 1979; Wu \& Kaiser, 1995). Pili are extended at one cell pole, attach to the extracellular polysaccharides (EPS) on the surface of the substratum or another cell, and retract to pull the cell forward (Li et al., 2003). TFP are composed of several thousand subunits of PilA or pilin. The cycles of TFP extension and retraction are achieved by assembling PilA into polar filaments with the assistance of the ATPase PilB (Jakovljevic et al., 2008), followed by disassembling the pili into single subunits during retraction, a process that is mediated by the ATPase PilT (Jakovljevic et al., 2008). PilA monomers form a membrane-associated pool which can be recycled to form new TFP (Yang et al., 2010).

Although the structure of $M$. xanthus PilA has not been experimentally elucidated, the crystal structures of closely related pilins in $P$. aeruginosa (PilA) and $N$. gonorrhoeae (PilE) have been solved either in their full-length or in their N-terminal-truncated forms (Craig et al., 2003, 2006; Hazes et al., 2000; Keizer et al., 2001; Parge et al., 1995). 
These studies have revealed a remarkably conserved structure for full-length pilin, which consists of an $\mathrm{N}$ terminal $\alpha$-helix domain followed by a C-terminal globular domain. Consistent with data derived from fibre diffraction and electron microscopy (Craig et al., 2006), a TFP assembly model has been proposed based on these crystal structures, in which pilins are suggested to assemble into the pilus in a helical manner. The $\mathrm{N}$-terminal $\alpha$-helix forms the hydrophobic core, and the C-terminal globular domain forms both lateral and longitudinal contacts at the outer surface (Craig et al., 2004). The N-terminal $\alpha$-helix is also believed to be essential for the membrane trafficking of PilA (Aas et al., 2007; Yang et al., 2010). This N-terminal domain can be further separated into a highly conserved hydrophobic $\alpha 1-\mathrm{N}$ subdomain (residues 13-40, counting from the first amino acid in the signal peptide) and a less conserved amphipathic $\alpha 1-\mathrm{C}$ subdomain (residues 41-65) (Craig et al., 2004). $\alpha 1-\mathrm{N}$ is predicted to mediate both membrane localization and crucial contacts between the subunits for TFP assembly. The $\mathrm{C}$ terminus of PilA has been shown to mediate cell attachment, and binding to surfaces and extracellular sugars (Craig et al., 2006; Giltner et al., 2006; Lee et al., 1994; Li et al., 2003; Scheuerpflug et al., 1999; Sheth et al., 1995).

The high degree of conservation observed among the PilA $\mathrm{N}$-terminal $\alpha$-helix domains from different bacteria ( $\mathrm{Li}$ et al., 2005) suggests that M. xanthus pilin may assemble into TFP in a manner similar to that predicted for $P$. aeruginosa and $N$. gonorrhoeae. Previously, we have shown that specific pilA sequence changes in $M$. xanthus can affect TFP assembly and PilA localization, which in turn alter EPS production and fruiting body formation (Yang et al., 2010). Additional roles of the PilA sequence and its structure-function relationship in TFP-dependent S motility still remain to be elucidated. In this study, we used a pilA mutant library (Yang et al., 2010) to isolate a mutant that produced TFP but failed to swarm on $0.3 \%$ agar (Shi \& Zusman, 1993). We subsequently mapped the mutation to A32 and generated additional single-residue mutant variants of PilA with alterations at this position for functional characterization. This allowed us to establish the critical role of amino acid A32 in PilA stability and TFP-related functions.

\section{METHODS}

Bacterial strains, plasmids and culture conditions. $M$. xanthus strains and plasmids used in this study are listed in Table 1. M. xanthus cells were grown in CYE medium $(1 \%$, w/v, casitone, $0.5 \%$, w/v, yeast extract, $8 \mathrm{mM} \mathrm{MgSO}_{4}$ in $10 \mathrm{mM}$ MOPS buffer, $\mathrm{pH}$ 7.6) (Campos et al., 1978 ) at $32{ }^{\circ} \mathrm{C}$ on a rotary shaker at 300 r.p.m. and were maintained on $1.5 \%$, w/v, agar plates. The Escherichia coli strain DH5 $\alpha$ used for cloning and plasmid construction was grown at $37{ }^{\circ} \mathrm{C}$ in Luria-Bertani medium (Sambrook \& Russell, 2001). Medium and plates were supplemented with kanamycin at $100 \mu \mathrm{g} \mathrm{ml}^{-1}$ when needed.

Construction of pilA mutants. Site-directed mutagenesis of pilA was conducted using two-step overlap PCR as previously described
(Yang et al., 2010) and adapted from the standard protocol (Sambrook \& Russell, 2001). The mutant pilA gene was cloned into the EcoRI and BamHI sites of pSWU19, and the resulting plasmids were electroporated (Kashefi \& Hartzell, 1995) into DK10410 ( $\Delta$ pilA) (Wu \& Kaiser, 1996) and SW2017 ( $\Delta$ pilA $\Delta$ difA) to select for kanamycin resistance. All plasmids and mutants constructed in this study were confirmed by PCR and sequencing (data not shown). The sequences of the primers used for mutant construction are shown in Table 2 .

Swarming assay. The swarming assay was performed as previously described (Shi \& Zusman, 1993). Briefly, M. xanthus cells were grown in CYE medium to the exponential growth phase and concentrated to $\mathrm{OD}_{600} 10\left(5 \times 10^{9}\right.$ cells $\left.\mathrm{ml}^{-1}\right)$ in CYE medium. An aliquot of $5 \mu \mathrm{l}$ concentrated culture was spotted onto $0.3 \%$ or $1.5 \%$ CYE agar plates and incubated for 3 days at $32{ }^{\circ} \mathrm{C}$. S motility was analysed by observing the colonies for expansion on $0.3 \%$ CYE agar plates.

Calcofluor white binding assay. EPS production was visualized using a calcofluor white binding assay as described by Black \& Yang (2004). Briefly, exponential growth phase cells were concentrated to $\mathrm{OD}_{600} 10$ in MOPS buffer (10 mM MOPS, $8 \mathrm{mM} \mathrm{MgSO}_{4}, \mathrm{pH} 7.6$ ). An aliquot of $5 \mu \mathrm{l}$ concentrated culture was spotted onto $0.3 \% \mathrm{CYE}$ agar plates containing calcofluor white (CF agar) at $50 \mu \mathrm{g} \mathrm{ml}^{-1}$. The plates were incubated at $32{ }^{\circ} \mathrm{C}$ in the dark for 3 days before they were examined and photographed under the illumination of a longwavelength (365 $\mathrm{nm}$ ) UV light source.

Fruiting body formation assay. M. xanthus cells were grown in CYE to exponential growth phase and concentrated to $\mathrm{OD}_{600} 10$ in Tris-phosphate-magnesium (TPM) buffer $(10 \mathrm{mM}$ Tris/ $\mathrm{HCl}$, $\left.\mathrm{pH} 7.6,1 \mathrm{mM} \mathrm{KH}{ }_{2} \mathrm{PO}_{4}, 8 \mathrm{mM} \mathrm{MgSO}{ }_{4}\right)$. Aliquots $(5 \mu \mathrm{l})$ of concentrated cells were spotted onto CF agar (Hagen et al., 1978) and incubated for 3 days at $32{ }^{\circ} \mathrm{C}$. Pictures of fruiting bodies were taken using a Nikon Eclipse TE200 inverted microscope fitted with a SPOT camera/software (Diagnostic Instruments).

Western blot analysis of whole-cell PilA and surface pili. Western blot analysis was performed as described previously (Yang et al., 2010) following standard protocols (Harlow \& Lane, 1988). For whole-cell lysates, $10^{8}$ M. xanthus cells were lysed by boiling in SDSPAGE loading buffer for $10 \mathrm{~min}$. For surface pili detection, TFP were sheared off from $10^{10} \mathrm{M}$. xanthus cells by vigorous vortexing for $20 \mathrm{~min}$. The suspension was sedimented at $16000 \mathrm{~g}$ for $5 \mathrm{~min}$ and the supernatant was transferred to a clean tube. Pili/pilin in the solution were precipitated by adding TCA to a final concentration of $10 \%$, incubating on ice overnight, and sedimenting at $16000 \mathrm{~g}$ for $30 \mathrm{~min}$ at $4{ }^{\circ} \mathrm{C}$. Pellets were washed once with acetone, resuspended in SDSPAGE loading buffer and boiled for $10 \mathrm{~min}$. Primary anti-PilA antibody ( $\mathrm{Li}$ et al., 2005) was used at a 1:10000 dilution and antirabbit horseradish-peroxidase-conjugated secondary antibody (Pierce) was used at a 1:20000 dilution. Blots were developed using the Supersignal West Pico chemiluminescence reagent (Pierce). Images were taken with the ChemiDoc XRS system (Bio-Rad).

Pilin precipitation assay and mixing assay. Pilin precipitation and mixing assays were performed as previously described ( $\mathrm{Li}$ et al., 2003). For pilin precipitation, cell surface pili were sheared off from $10^{10}$ cells by vortexing as described above. The isolated pili were incubated with chitin suspension (final concentration $0.5 \mathrm{mg} \mathrm{ml}^{-1}$ ) or purified EPS $\left(0.5 \mathrm{mg} \mathrm{ml}^{-1}\right.$ carbohydrate) at $32{ }^{\circ} \mathrm{C}$ for $30 \mathrm{~min}$. The mixtures were pelleted by centrifugation at $6000 \mathrm{~g}$ for $5 \mathrm{~min}$ for chitin and $10000 \mathrm{~g}$ for $10 \mathrm{~min}$ for EPS. Supernatants were discarded; pellets were resuspended in $80 \mu \mathrm{l}$ SDS-PAGE loading buffer and boiled for $10 \mathrm{~min}$ before analysis by Western blotting. For the mixing/ retraction assay, EPS and chitin suspensions were mixed with $5 \times 10^{9}$ 
Table 1. Strains and plasmids used in this study

\begin{tabular}{|c|c|c|}
\hline Strain or plasmid & Relevant genotype & Reference or source \\
\hline \multicolumn{3}{|l|}{ Strains } \\
\hline DK1622 & Wild-type & Kaiser (1979) \\
\hline DK10410 & $\Delta p i l A$ & Wu \& Kaiser (1996) \\
\hline DK10409 & $\Delta p i l T$ & Wu et al. (1997) \\
\hline SW504 & $\Delta$ difA & Yang et al. (1998) \\
\hline SW2030 & pilA-A32G & This study \\
\hline SW2031 & pilA-A32V & This study \\
\hline SW2032 & pilA-A32L & This study \\
\hline SW2033 & pilA-A32S & This study \\
\hline SW2034 & pilA-A32P & This study \\
\hline SW2035 & pilA-A32K & This study \\
\hline SW2036 & pilA-A32D & This study \\
\hline SW2037 & pilA-A32E & This study \\
\hline SW2038 & pilA-A30P & This study \\
\hline SW2039 & pilA-A31P & This study \\
\hline SW2040 & pilA-A33P & This study \\
\hline SW2041 & pilA-P34A & This study \\
\hline SW2042 & pilA-A32V, difA & This study \\
\hline \multicolumn{3}{|l|}{ Plasmids } \\
\hline pSWU19 & Cloning vector with $\mathrm{Mx} 8$ attP locus for integration into $M$. xanthus genome, $\mathrm{Km}^{\mathrm{R}}$ & Wu \& Kaiser (1995) \\
\hline pSWU359 & pSWU19 carrying the 872 bp pilA gene and its promoter region, $\mathrm{Km}^{\mathrm{R}}$ & Wu \& Kaiser (1996) \\
\hline pMX08 & pilA-A32G in pSWU19 & This study \\
\hline pMX09 & pilA-A32V in pSWU19 & This study \\
\hline pMX10 & pilA-A32L in pSWU19 & This study \\
\hline pMX11 & pilA-A32S in pSWU19 & This study \\
\hline pMX12 & pilA-A32P in pSWU19 & This study \\
\hline pMX13 & pilA-A32K in pSWU19 & This study \\
\hline pMX14 & pilA-A32D in pSWU19 & This study \\
\hline pMX15 & pilA-A32E in pSWU19 & This study \\
\hline pMX16 & pilA-A30P in pSWU19 & This study \\
\hline pMX17 & pilA-A31P in pSWU19 & This study \\
\hline pMX18 & pilA-A33P in pSWU19 & This study \\
\hline pMX19 & pilA-P34A in pSWU19 & This study \\
\hline
\end{tabular}

cells and incubated at $32{ }^{\circ} \mathrm{C}$ for $30 \mathrm{~min}$. Remaining cell surface pili were analysed by Western blotting.

Tethering assay. To test for tethering of M. xanthus to solid surfaces via TFP, video microscopy assays were conducted as described previously (Sun et al., 2000). M. xanthus cells at the mid-exponential growth phase were serial diluted in MOPS buffer to obtain concentrations of about 50 isolated cells in the microscope field of view. Aliquots $(10 \mu \mathrm{l})$ of each sample were deposited into each well of a 24 -well cell culture plate containing $1 \%$, w/v, methylcellulose solution in MOPS buffer. Plates were incubated at $32{ }^{\circ} \mathrm{C}$ for $30 \mathrm{~min}$ or longer to allow cells to settle on the bottom of the well. Gliding motility and tethering behaviour were observed using an inverted microscope (Nikon) with a $\times 40$ objective lens. Serial digital images were taken at $5 \mathrm{~s}$ intervals using a Spot camera (Diagnostic Instruments).

Electron microscopy. Exponentially growing $M$. xanthus cells were washed once with MOPS buffer, stained using $2.5 \%$ uranyl acetate and examined with a CM120 transmission electron microscope (FEI) operated at $120 \mathrm{kV}$ with a LaB6 filament. Images were recorded with a Tietz F224HD charge-coupled device (CCD) camera with the EMMENU4 digital camera software. Images were taken at $\times 13000$ magnification.

\section{RESULTS}

\section{PilA mutant A32V produces TFP but is unable to swarm}

By screening a previously generated $M$. xanthus pilA mutant library (Yang et al., 2010), one mutant was identified that was deficient in S motility, despite its ability to produce surface pili. This mutant, which was designated A32V (Fig. 1a), exhibited impaired S motility when its swarming zones were compared with those of the wild-type strain on $0.3 \%$ agar (Fig. 1b). However, in contrast to the smooth edge produced by $\Delta p i l A$, the colony edge of mutant $\mathrm{A} 32 \mathrm{~V}$ was rough, indicating residual S motility. As visualized by the calcofluor white binding assay (Black \& Yang, 2004), A32V was found to produce detectable amounts of EPS and retain the ability to form fruiting bodies (Fig. 1b). Since surface pili act as a positive signal for EPS production (Black et al., 2006) and A32V produces more EPS than $\Delta$ pilA (Fig. 1b), we speculated that this mutant may still possess surface pili. Western blot analysis 
Table 2. Primers used in this study

\begin{tabular}{|c|c|}
\hline Primer used for mutant construction & Sequence $\left(5^{\prime}-3^{\prime}\right)$ \\
\hline 3' pilA flanking & GGCCTCTTCGCTATTACGC \\
\hline A32G-downstream & CTGGCCGCCATCGGCATCCCGAACTTC \\
\hline A32V-upstream & GAAGTTCGGGATGACGATGGCGGCCAG \\
\hline A32V-downstream & CTGGCCGCCATCGTCATCCCGAACTTC \\
\hline A32L-downstream & GGCATCCTGGCCGCCATCCTAATCCCGAACTTCATCAAG \\
\hline A32S-upstream & CTTGATGAAGTTCGGGATGCTGATGGCGGCCAGGATGCCG \\
\hline A32S-downstream & CGGCATCCTGGCCGCCATCAGCATCCCGAACTTCATCAAG \\
\hline A32P-upstream & CTTGATGAAGTTCGGGATTGGGATGGCGGCCAGGATGCCG \\
\hline A32P-downstream & CGGCATCCTGGCCGCCATCCCAATCCCGAACTTCATCAAG \\
\hline A32K-upstream & CTTGATGAAGTTCGGGATCTTGATGGCGGCCAGGATGCCG \\
\hline A32E-downstream & CGGCATCCTGGCCGCCATCGAAATCCCGAACTTCATCAAG \\
\hline A30P-upstream & GAAGTTCGGGATGGCGATGGGGGCCAGGATGCCGATGATG \\
\hline A30P-downstream & CATCATCGGCATCCTGGCCCCCATCGCCATCCCGAACTTC \\
\hline A31P-upstream & GATGAAGTTCGGGATGGCGGGGGCGGCCAGGATGCCGATG \\
\hline A31P-downstream & CATCGGCATCCTGGCCGCCCCCGCCATCCCGAACTTCATC \\
\hline A33P-upstream & GAACTTGATGAAGTTCGGTGGGGCGATGGCGGCCAGGATG \\
\hline A33P-downstream & CATCCTGGCCGCCATCGCCCCACCGAACTTCATCAAGTTC \\
\hline P34A-upstream & CTTGATGAAGTTCGCGATGGCGATGGCGG \\
\hline P34A-downstream & CCGCCATCGCCATCGCGAACTTCATCAAG \\
\hline
\end{tabular}

revealed that whole-cell PilA expression levels in A32V were similar to those in the wild-type, while the amount of surface pili appeared to be elevated in the mutant strain (Fig. 1c). Electron microscopy confirmed the existence of TFP on the A32V cell surface (Fig. 1d). Based on these results, we hypothesize that the PilA in $\mathrm{A} 32 \mathrm{~V}$ can assemble into surface pili but are unable to retract. Comparison of A32V with a $\Delta$ pilT mutant strain that is defective in the $M$. xanthus ATPase responsible for TFP retraction (Nudleman \& Kaiser, 2004; Wu et al., 1997) showed that both strains were similarly impaired in S motility, had rough colony edges/surfaces and produced EPS (Fig. 1b). However, in $\Delta p i l T$, the amount of whole-cell PilA was significantly higher than in both the wild-type and $\mathrm{A} 32 \mathrm{~V}$, and more pili were present on the cell surface (Fig. 1c).

\section{A32V TFP has EPS-binding ability but is unable to retract}

The apparent overpiliation and $S$ motility-deficient phenotypes in $\mathrm{A} 32 \mathrm{~V}$ suggested that the mutated PilA is capable of assembling into TFP that are unable to retract. This could be due to two different mechanisms. First, the mutagenized TFP in A32V may have lost the ability to bind EPS and is therefore unable to recognize the retraction trigger (Li et al., 2003); second, the TFP in A32V still bind to their target EPS but are unable to retract. To distinguish between these two possibilities, PilA-A32V was introduced into a $\Delta$ pilA $\Delta$ difA double mutation background. In the $\Delta$ difA background, all assembled pili remain on the cell surface because the cells are defective in EPS production and therefore lack the trigger for retraction (Yang et al., 1998, 2000). The addition of purified EPS from wild-type cells or the EPS analogue chitin was found to trigger TFP retraction and abolish the overpiliation phenotype (Li et al., 2003). Using the $\Delta$ difA mutant as a control, the pilA-A32V $\Delta$ difA mutant allowed us to verify TFP assembly in A32V and to test the binding and retracting ability of the mutated TFP. Pili were purified from the cell surface by shearing and were analysed using Western blots. pilA-A32V $\Delta$ difA produced amounts of surface pili similar to $\Delta$ difA (Fig. 2a, lane 1), demonstrating that the mutated $\mathrm{A} 32 \mathrm{~V}$ pilin can assemble into TFP in the $\Delta$ difA background.

To test the binding ability of the TFP, sheared pili were incubated with either chitin (Fig. 2a, lane 2) or EPS isolated from the wild-type strain DK1622 (Fig. 2a, lane 3) and subjected to the precipitation assay. Similar to wild-type pili, the mutated A32V pili were precipitated by EPS and chitin at the same levels, indicating that the mutated TFP still have binding ability. To test if the mutated TFP can retract, cells were subjected to the mixing assay to trigger TFP retraction using either chitin (Fig. 2a, lane 5) or EPS (Fig. 2a, lane 6). While $\Delta$ difA retracted its pili after incubation with both chitin and EPS, the pilA-A32V $\Delta$ difA mutant showed little reduction in recoverable surface TFP, 


\section{(a) MRVSRFNPRNRGFTLIELMIVVAIIGILAAIAIPNFIKFOARSKOSEAKTNLKALYTAOK 60 SFFSEKDRYSDFANEIGFAPERGNRYGYRVSAAAGDCEVRNAADLPVPAAGVPCISNDSF 120 RFGANSAIDDPTPVVARFVPQGAAGWNTTLGVQPTIADCPNCNFFAGARGNADNEATFDD 180 WVIAGFEGSGQVGPCSEAGNVASGTPYNTRNDVACDGAAQ 220}

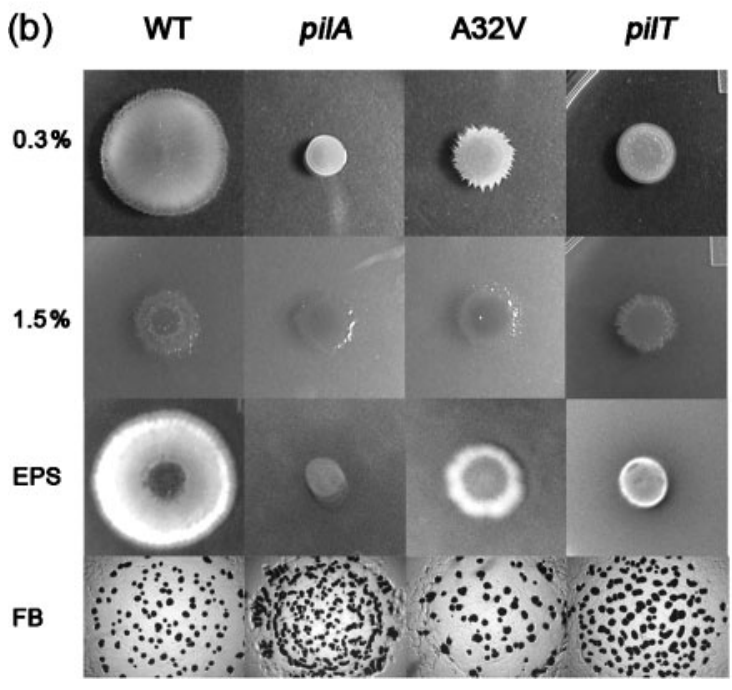

(c)

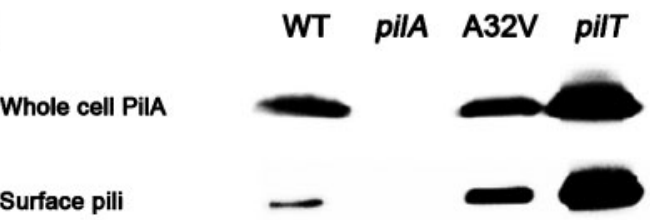

(d)

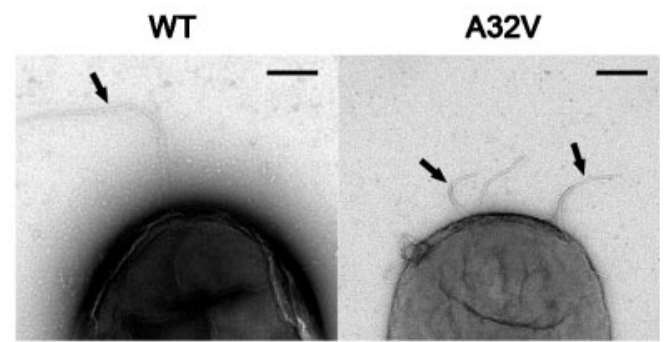

Fig. 1. Phenotype summary of the PilA mutant A32V. (a) PilA amino acid sequence in the $M$. xanthus wild-type strain. Grey type shows the signal peptide of PilA; underlined sequences correspond to the $\mathrm{N}$-terminal $\alpha$-helix predicted using the SCRATCH protein predictor (http://scratch.proteomics.ics.uci.edu/). The residue marked with a dot underneath is alanine 32. (b) Motility, surface properties and social phenotypes of mutant A32V. Top to bottom rows show swarming on $0.3 \%$ agar surfaces, swarming on $1.5 \%$ agar surfaces, EPS production and fruiting body (FB) formation. Strains from left to right are wild-type strain DK1622 (WT), $\triangle$ pilA, mutant A32V and $\Delta$ pilT. All experiments were repeated three times and representative images are shown. (c) Whole-cell PilA production and surface pili were tested using Western blots. The same four strains were used as in panel (b). (d) Electron microscopy images showing wild-type and A32V cell surface pili (indicated by black arrows). Bar, $200 \mathrm{~nm}$.

indicating that retraction of TFP was not triggered by the presence of EPS or chitin. Therefore, the mutated A32V pilin/pili still recognized its/their target polysaccharides although the mutated TFP were unable to retract.

To further confirm our findings, a video microscopy-based assay was used to provide direct visual evidence that the mutant $\mathrm{A} 32 \mathrm{~V}$ pili still have binding ability but are unable to retract. Sun et al. (2000) developed an assay capable of measuring $S$ motility at the single-cell level. When deposited in $1 \%$ methylcellulose in MOPS buffer on polystyrene surfaces, wild-type M. xanthus cells glide over the surface and occasionally tether to the surface with their TFP, resulting in the detection of cells that 'stand up' (Fig. $2 \mathrm{~b})$. Cells lacking TFP ( $\triangle$ pilA) were non-motile in this assay and unable to 'stand up'. We analysed $500 \mathrm{~A} 32 \mathrm{~V}$ mutant cells under these experimental conditions and did not find motile cells; however, we did observe tethering behaviour (Fig. 2b). The phenotype of A32V in this assay is similar to that of the TFP retraction-deficient mutant pilT (Sun et al., 2000). These results further confirmed that $\mathrm{A} 32 \mathrm{~V}$ produces TFP, which allows the cells to tether, although the pili are unable to retract. As a consequence, $S$ motility is impaired in the mutant strain.

\section{Site-specific mutations at amino acid 32 of PilA result in mutants with different phenotypes}

The only mutation in $\mathrm{A} 32 \mathrm{~V}$ is the alanine to valine mutation at position 32 in the $\alpha$-helix of PilA, indicating the importance of this amino acid for PilA function. To further investigate the role of A32, we conducted sitespecific mutagenesis of pilA to obtain a series of singleresidue variants at the same position in PilA. The original alanine was changed to amino acids with different $\mathrm{R}$ groups (side chains) and different chemical properties (Mathews et al., 2000), resulting in A32G, A32V, A32L, A32S, A32P, A32K, A32D and A32E. All mutants were tested for $S$ motility, EPS production, fruiting body formation, PilA production and surface TFP assembly. Changing alanine into amino acids within the same $\mathrm{R}$ group (A32G, A32V, A32L) or into another structurally similar and uncharged residue (A32S) resulted in PilA production at wild-type levels (Fig. 3). Replacement of 
(a)
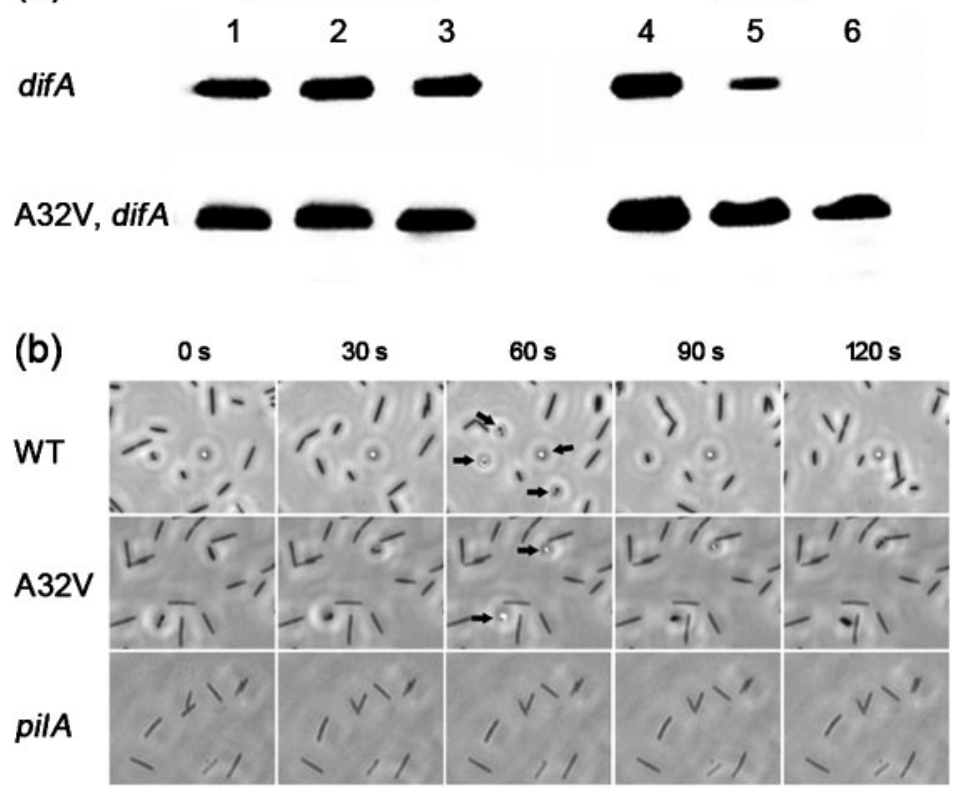

Fig. 2. TFP in $A 32 V$ have binding ability but are unable to retract. (a) TFP binding and retraction abilities were tested using precipitation and mixing assays. For precipitation, TFP were sheared off from the cell surface of difA and the pilA-A32V, difA double mutant and precipitated with either chitin or wild-type EPS. Lanes: 1, total sheared pili; 2, precipitated by chitin; 3, precipitated by EPS. Mixing/ retraction assays were conducted by preincubating cells with MOPS buffer, chitin or EPS for $30 \mathrm{~min}$, followed by vigorous shearing to measure the remaining surface pili. Lanes: 4 , cells pre-incubated with MOPS buffer; 5 , cells pre-incubated with chitin; 6, cells pre-incubated with EPS. (b) Visual evidence that A32V TFP can tether to polystyrene surfaces. $M$. xanthus cells were deposited into 24-well cell culture plates containing $1 \%$ methylcellulose in MOPS buffer, and individual cells were analysed for motility and tethering behaviour on solid surfaces. Tethered cells appear as dots in the image, indicated by black arrows. Top to bottom photos show the wild-type strain DK1622 (WT), A32V and $\Delta$ pilA. Left to right images were taken at $30 \mathrm{~s}$ intervals, showing that cells with TFP can change between tethering and 'lying down' status. alanine with negatively or positively charged amino acids (A32K, A32D, A32E) or proline (A32P) led to a dramatic reduction in PilA levels (Fig. 3). For the mutants with little or no PilA expression, no surface pili were detected and little EPS were observed in the calcofluor white binding assay. Of the mutants that still produced wild-type levels of PilA, the surface pili of A32S were similar to those of the wild-type. Interestingly, however, the mutants A32G, A32V and $\mathrm{A} 32 \mathrm{~L}$ exhibited progressive phenotypes corresponding to the increase in side-chain length of the R group. Glycine $(G)$ is one methyl group shorter than alanine, and the phenotype of $\mathrm{A} 32 \mathrm{G}$ was similar to that of the wild-type. For this mutant, the same amount of surface pili was detected, and the TFP were fully functional, as indicated by its ability to swarm on $0.3 \%$ agar. In the mutant PilA of $\mathrm{A} 32 \mathrm{~V}$, alanine at position 32 is replaced by valine $(\mathrm{V})$, which carries a side chain that is two methyl groups longer than the original alanine. PiA-A32V was able to assemble into TFP, although the mutated TFP was not able to retract, as indicated by the lack of detectable swarming despite the presence of EPS (Fig. 3). Extension of the side chain to leucine in PilA-A32L resulted in a more dramatic phenotype. Although A32L produced whole-cell PilA at wild-type levels, no surface pili were detected. The structural changes in A32L PilA may impair the ability of the mutated pilin to assemble into TFP. Therefore, PilA monomers in A32L accumulated in the cell, likely in the membrane, leading to dramatic EPS reductions and a nonfruiting phenotype (Fig. 3) (Yang et al., 2010). These findings for A32 variants demonstrated that the uncharged nature of A32 is important for the stability of PilA, while side-chain length is important for the proper assembly of pilin subunits into functional pili.

\section{Proline mutations in the $\alpha$-helix of PilA result in mutants with unstable PilA}

The PilA sequence contains a proline at residue 34 that is close to A32 and is believed to be essential for the formation of a kink in the $\alpha 1-\mathrm{N}$ subdomain (Craig et al., 2006) (Fig. 1a). The curved structure created by this kink has been implicated as assisting in the tight packing of pilin subunits into TFP. It is possible that the phenotypes we observed in the A32 mutants were due to structural changes affecting this kink. In order to verify the importance of P34 in PilA, we replaced P34 with alanine. In the resulting mutant, P34A, both whole-cell PilA and surface pili were expressed at reduced levels compared with the wild-type and resulted in a corresponding reduction in S motility on $0.3 \%$ agar (Fig. 4). These results indicated that although $\mathrm{P} 34 \mathrm{~A}$ produced less pilin, the mutated pilins still assembled into functional TFP. Therefore, the absence of the kink due to substitution of the proline with alanine affected PilA production but not TFP assembly or its function. To further study the role of the $\alpha$-helix structure in PilA, we generated three additional mutants by replacing amino acids near the kink region with prolines (A30P, I31P, I33P). These three proline mutants showed pheno- 

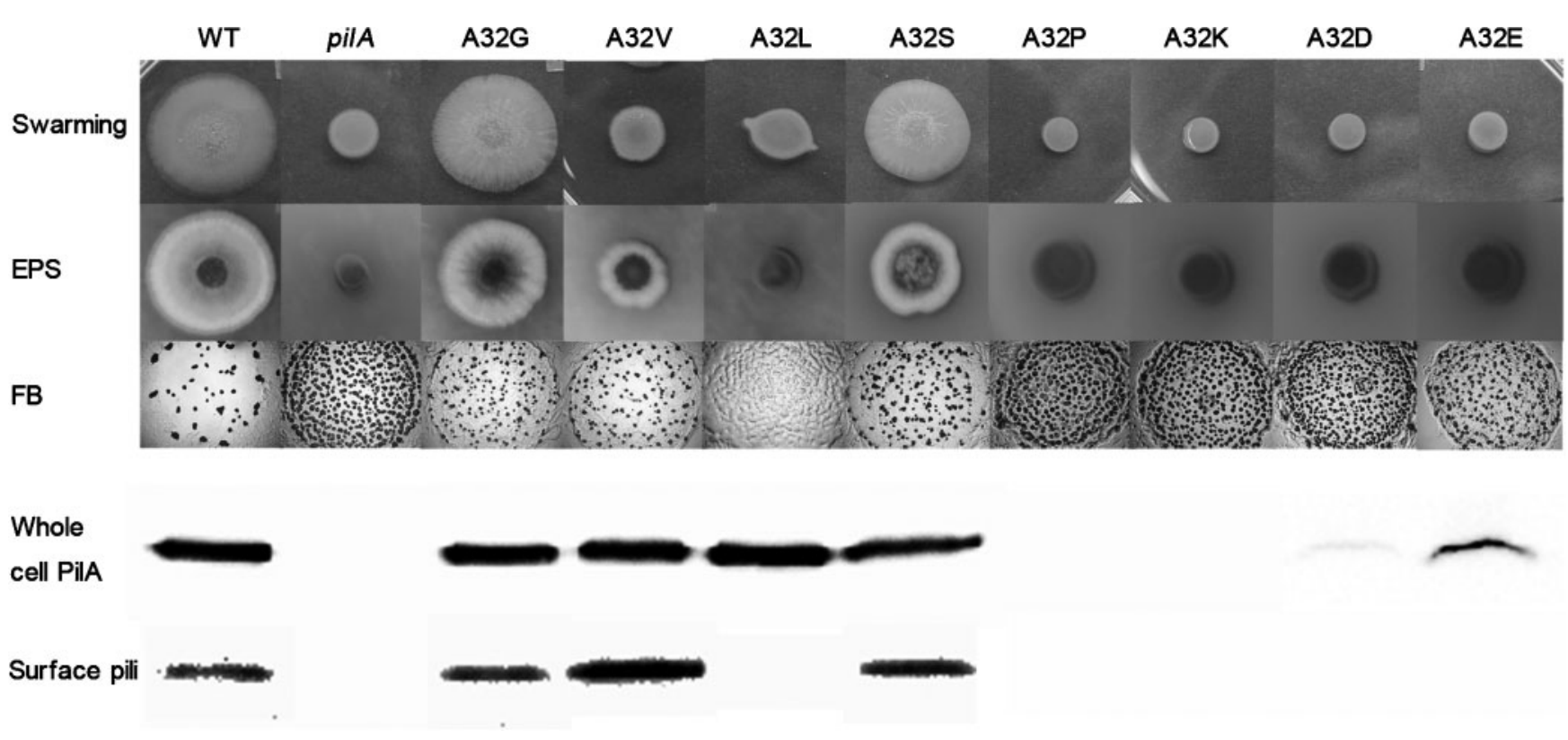

Fig. 3. Effects of PilA A32 mutations on TFP production and function. Eight M. xanthus mutants with different site-specific mutations at PilA A32 were tested for their effects on TFP-related phenotypes. Top to bottom rows show swarming ability on a $0.3 \%$ agar surface, EPS production, fruiting body (FB) formation, whole-cell PilA production and surface pili production. Western blot data for the whole-cell PilA and surface pili were reorganized from two original blots. WT, wild-type.

types similar to that of A32P in that none of the mutants produced PilA or EPS, and no swarming was detected on $0.3 \%$ agar (Fig. 4). These data demonstrated that dramatic structural changes such as a turning produced by a proline residue in the $\alpha 1-\mathrm{N}$ subdomain of PilA are not tolerated and may lead to instability of PilA in M. xanthus.

\section{DISCUSSION}

In this study, we characterized a pilA mutant that was deficient in S motility despite its ability to produce surface pili. The mutation mapped to A32 of PilA, which was found to be important in a previous study (Yang et al.,

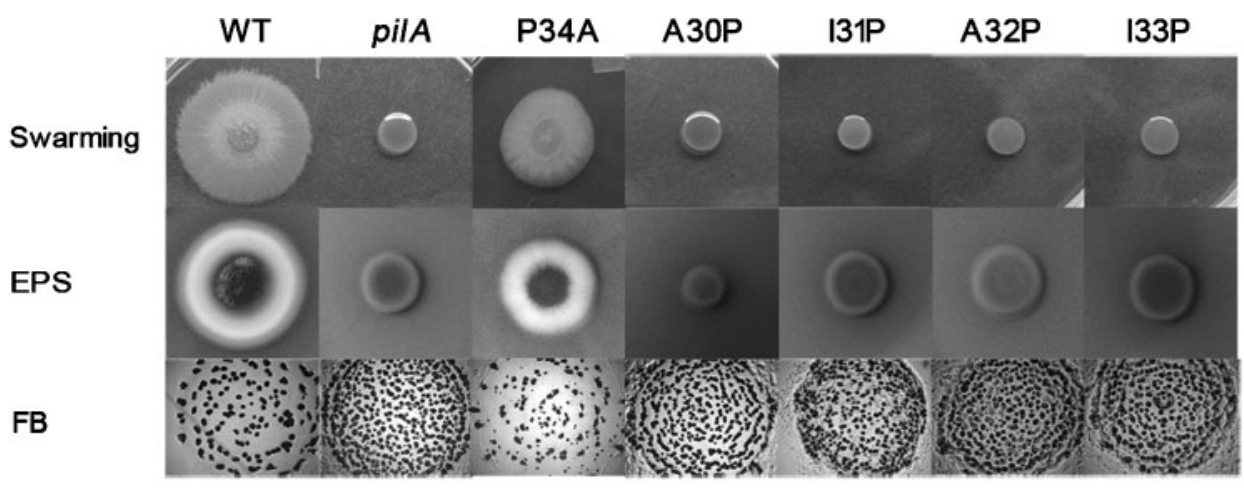

Whole

cell PilA

Surface pili

Fig. 4. Proline mutations in the $\alpha$-helix of PilA result in mutants with unstable PilA. Four M. xanthus mutants with proline mutations in the $\alpha$-helix of PilA were tested for their PilA-related phenotypes. Top to bottom rows show swarming ability on a $0.3 \%$ agar surface, EPS production, fruiting body (FB) formation, whole-cell PilA production and surface pili production. Western blot data for the whole-cell PilA and surface pili were reorganized from two original blots. WT, wild-type. 
2010). By further characterizing this mutant as well as additional pilA point mutants at this position, we concluded that A32 is important for PilA stability, TFP assembly and related functions. We also showed that different amino acid structures at position 32 in PilA have different effects on these features.

Through genetic, biochemical and video microscopic assays, we found that PilA-A32V is produced at wild-type levels and assembles into TFP (Fig. 1). These TFP, however, are unable to retract. In the TFP assembly model, the Nterminal $\alpha$-helix of PilA is packed in the TFP core, where it interacts via both hydrophobic and electrostatic interactions (Craig et al., 2006). The $\alpha 1-\mathrm{N}$ subdomain (amino acids 13-40) is almost entirely hydrophobic and thus provides an optimal environment for tight packing. In addition, the negatively charged residue $17 \mathrm{E}$ and positively charged $\mathrm{N}$ terminus of residue $13 \mathrm{~F}$ point toward the filament centre and form an inter-subunit salt bridge which strengthens the binding between pilin subunits. The sequence of this PilA subdomain is optimized to maximize the contacts between neighbouring subunits. The valine replacement at the alanine 32 site may have altered the structure of the $\alpha 1-\mathrm{N}$ subdomain of PilA and consequently altered the inter-subunit interaction. This possible structural change in the assembled TFP could then interfere with the retraction process.

How do structural changes in A32V TFP affect retraction and $\mathrm{S}$ motility? We suggest the following three possibilities that would be consistent with the increased level of surface pili detected using Western blotting in the TFP shear-off assay (Fig. 1c) and the lack of S motility (Figs $1 \mathrm{~b}$ and $2 \mathrm{~b}$ ). (1) The stability of the mutated TFP is reduced due to the lack of tight packing and close interactions at the core of the TFP filament. Although PilA-A32V can be assembled into TFP by PilB, the reduced stability of the mutated TFP leads to filament disintegration upon the pulling force exerted during the PilT-mediated retraction process. The pili would break apart before the cells move forward, leading to a drastic reduction in $S$ motility. (2) The alterations in PilA affect its interaction with the retraction motor PilT. PilT forms hexameric polymers (Satyshur et al., 2007) that are essential for TFP retraction, which is powered by the ATPase activity of PilT (Jakovljevic et al., 2008). The structural changes in the mutated TFP may prevent correct interaction of PilA with PilT or affect the force between TFP and the PilT machinery necessary for retraction. Therefore, retraction through the PilT apparatus is impaired. Initially we hypothesized that the mutated TFP may change the turning angle in the kink region of the $\alpha$ helix and thus affect the structure of the TFP and the interaction with PilT. However, the P34A mutant constructed in this study showed substantial S motility (Fig. 4), demonstrating that the kink in the $\alpha$-helix of PilA is less likely to be essential for TFP retraction. (3) Alterations in TFP affect signal transduction. TFP act as sensors for EPS production (Black et al., 2006), and the binding of EPS to TFP triggers pilus retraction (Li et al., 2003). Although the mutated TFP produced by $\mathrm{A} 32 \mathrm{~V}$ can still bind to EPS, the downstream signal transduction pathway to trigger the retraction process may be defective. Even if A32V does not totally block the retraction signal, it is possible that the altered TFP produce a weakened signal for retraction which may alter the balance between extension and retraction, leading to a net increase in extension.

The $\alpha$-helix, especially the $\alpha 1-\mathrm{N}$ domain in PilA, is highly conserved among different bacteria, including non-pathogenic and pathogenic species such as $P$. aeruginosa and $N$. gonorrhoeae. Our findings for M. xanthus PilA will aid in our understanding of the minimal structural requirements for successful assembly of TFP and shed light on the structure-function relationship between PilA and TFPdependent motility. Finally, further studies to solve the detailed structure of M. xanthus PilA are needed in order to better understand the complexity of TFP.

\section{ACKNOWLEDGEMENTS}

This work was supported by the National Institutes of Health (NIH) grant GM54666 to W.S. We acknowledge the use of cryoEM facilities at the Electron Imaging Center for NanoMachines supported in part by the NIH (1S10RR23057 to Z.H.Z.).

\section{REFERENCES}

Aas, F. E., Winther-Larsen, H. C., Wolfgang, M., Frye, S., Løvold, C., Roos, N., van Putten, J. P. \& Koomey, M. (2007). Substitutions in the $\mathrm{N}$-terminal alpha helical spine of Neisseria gonorrhoeae pilin affect type IV pilus assembly, dynamics and associated functions. Mol Microbiol 63, 69-85.

Black, W. P. \& Yang, Z. (2004). Myxococcus xanthus chemotaxis homologs DifD and DifG negatively regulate fibril polysaccharide production. J Bacteriol 186, 1001-1008.

Black, W. P., Xu, Q. \& Yang, Z. (2006). Type IV pili function upstream of the Dif chemotaxis pathway in Myxococcus xanthus EPS regulation. Mol Microbiol 61, 447-456.

Bradley, D. E. (1980). A function of Pseudomonas aeruginosa PAO polar pili: twitching motility. Can J Microbiol 26, 146-154.

Campos, J. M., Geisselsoder, J. \& Zusman, D. R. (1978). Isolation of bacteriophage MX4, a generalized transducing phage for Myxococcus xanthus. J Mol Biol 119, 167-178.

Craig, L., Taylor, R. K., Pique, M. E., Adair, B. D., Arvai, A. S., Singh, M., Lloyd, S. J., Shin, D. S., Getzoff, E. D. \& other authors (2003). Type IV pilin structure and assembly: X-ray and EM analyses of Vibrio cholerae toxin-coregulated pilus and Pseudomonas aeruginosa PAK pilin. Mol Cell 11, 1139-1150.

Craig, L., Pique, M. E. \& Tainer, J. A. (2004). Type IV pilus structure and bacterial pathogenicity. Nat Rev Microbiol 2, 363-378.

Craig, L., Volkmann, N., Arvai, A. S., Pique, M. E., Yeager, M., Egelman, E. H. \& Tainer, J. A. (2006). Type IV pilus structure by cryoelectron microscopy and crystallography: implications for pilus assembly and functions. Mol Cell 23, 651-662.

Giltner, C. L., van Schaik, E. J., Audette, G. F., Kao, D., Hodges, R. S., Hassett, D. J. \& Irvin, R. T. (2006). The Pseudomonas aeruginosa type IV pilin receptor binding domain functions as an adhesin for both biotic and abiotic surfaces. Mol Microbiol 59, 1083-1096. 
Hagen, D. C., Bretscher, A. P. \& Kaiser, D. (1978). Synergism between morphogenetic mutants of Myxococcus xanthus. Dev Biol 64, 284-296.

Harlow, E. \& Lane, D. (1988). Antibodies: a Laboratory Manual. Cold Spring Harbor, NY: Cold Spring Harbor Laboratory.

Hazes, B., Sastry, P. A., Hayakawa, K., Read, R. J. \& Irvin, R. T. (2000). Crystal structure of Pseudomonas aeruginosa PAK pilin suggests a main-chain-dominated mode of receptor binding. J Mol Biol 299, 1005-1017.

Hodgkin, J. \& Kaiser, D. (1979). Genetics of gliding motility in Myxococcus xanthus (Myxobacterales): two gene systems control movement. Mol Gen Genet 171, 177-191.

Jakovljevic, V., Leonardy, S., Hoppert, M. \& Søgaard-Andersen, L. (2008). PilB and PilT are ATPases acting antagonistically in type IV pilus function in Myxococcus xanthus. J Bacteriol 190, 2411-2421.

Kaiser, D. (1979). Social gliding is correlated with the presence of pili in Myxococcus xanthus. Proc Natl Acad Sci U S A 76, 5952-5956.

Kashefi, K. \& Hartzell, P. L. (1995). Genetic suppression and phenotypic masking of a Myxococcus xanthus frzF $F^{-}$defect. Mol Microbiol 15, 483-494.

Keizer, D. W., Slupsky, C. M., Kalisiak, M., Campbell, A. P., Crump, M. P., Sastry, P. A., Hazes, B., Irvin, R. T. \& Sykes, B. D. (2001). Structure of a pilin monomer from Pseudomonas aeruginosa: implications for the assembly of pili. J Biol Chem 276, 24186-24193.

Lee, K. K., Sheth, H. B., Wong, W. Y., Sherburne, R., Paranchych, W., Hodges, R. S., Lingwood, C. A., Krivan, H. \& Irvin, R. T. (1994). The binding of Pseudomonas aeruginosa pili to glycosphingolipids is a tipassociated event involving the C-terminal region of the structural pilin subunit. Mol Microbiol 11, 705-713.

Li, Y., Sun, H., Ma, X., Lu, A., Lux, R., Zusman, D. \& Shi, W. (2003). Extracellular polysaccharides mediate pilus retraction during social motility of Myxococcus xanthus. Proc Natl Acad Sci U S A 100, 5443-5448.

Li, Y., Lux, R., Pelling, A. E., Gimzewski, J. K. \& Shi, W. (2005). Analysis of type IV pilus and its associated motility in Myxococcus xanthus using an antibody reactive with native pilin and pili. Microbiology 151, 353-360.

MacNeil, S. D., Mouzeyan, A. \& Hartzell, P. L. (1994). Genes required for both gliding motility and development in Myxococcus xanthus. Mol Microbiol 14, 785-795.

Mathews, C. K., Van Holde, K. E. \& Ahern, K. G. (2000). Biochemistry, 3rd edn. San Francisco, CA: Benjamin Cummings.

Nudleman, E. \& Kaiser, D. (2004). Pulling together with type IV pili. J Mol Microbiol Biotechnol 7, 52-62.

Parge, H. E., Forest, K. T., Hickey, M. J., Christensen, D. A., Getzoff, E. D. \& Tainer, J. A. (1995). Structure of the fibre-forming protein pilin at $2.6 \AA$ resolution. Nature 378, 32-38.
Sambrook, J. \& Russell, D. W. (2001). Molecular Cloning: a Laboratory Manual. Cold Spring Harbor, NY: Cold Spring Harbor Laboratory.

Satyshur, K. A., Worzalla, G. A., Meyer, L. S., Heiniger, E. K., Aukema, K. G., Misic, A. M. \& Forest, K. T. (2007). Crystal structures of the pilus retraction motor PilT suggest large domain movements and subunit cooperation drive motility. Structure 15, 363-376.

Scheuerpflug, I., Rudel, T., Ryll, R., Pandit, J. \& Meyer, T. F. (1999). Roles of PilC and PilE proteins in pilus-mediated adherence of Neisseria gonorrhoeae and Neisseria meningitidis to human erythrocytes and endothelial and epithelial cells. Infect Immun 67, 834843.

Sheth, H. B., Glasier, L. M., Ellert, N. W., Cachia, P., Kohn, W., Lee, K. K., Paranchych, W., Hodges, R. S. \& Irvin, R. T. (1995). Development of an anti-adhesive vaccine for Pseudomonas aeruginosa targeting the C-terminal region of the pilin structural protein. Biomed Pept Proteins Nucleic Acids 1, 141-148.

Shi, W. \& Zusman, D. R. (1993). The two motility systems of Myxococcus xanthus show different selective advantages on various surfaces. Proc Natl Acad Sci U S A 90, 3378-3382.

Sun, H., Zusman, D. R. \& Shi, W. (2000). Type IV pilus of Myxococcus xanthus is a motility apparatus controlled by the frz chemosensory system. Curr Biol 10, 1143-1146.

Wu, S. S. \& Kaiser, D. (1995). Genetic and functional evidence that type IV pili are required for social gliding motility in Myxococcus xanthus. Mol Microbiol 18, 547-558.

Wu, S. S. \& Kaiser, D. (1996). Markerless deletions of pil genes in Myxococcus xanthus generated by counterselection with the Bacillus subtilis sacB gene. J Bacteriol 178, 5817-5821.

Wu, S. S., Wu, J. \& Kaiser, D. (1997). The Myxococcus xanthus pilT locus is required for social gliding motility although pili are still produced. Mol Microbiol 23, 109-121.

Yang, Z., Geng, Y., Xu, D., Kaplan, H. B. \& Shi, W. (1998). A new set of chemotaxis homologues is essential for Myxococcus xanthus social motility. Mol Microbiol 30, 1123-1130.

Yang, Z., Ma, X., Tong, L., Kaplan, H. B., Shimkets, L. J. \& Shi, W. (2000). Myxococcus xanthus dif genes are required for biogenesis of cell surface fibrils essential for social gliding motility. J Bacteriol 182, 5793-5798.

Yang, Z., Lux, R., Hu, W., Hu, C. \& Shi, W. (2010). PilA localization affects extracellular polysaccharide production and fruiting body formation in Myxococcus xanthus. Mol Microbiol 76, 15001513.

Edited by: J. G. Shaw 